\title{
Differences in the mode of the extension of gastric cancer classified by histological type: new histological classification of gastric carcinoma
}

\author{
N Goseki, T Takizawa, M Koike
}

\begin{abstract}
By combining two of the morphological characteristics of gastric cancer, the degree of differentiation of the glandular tubules and the amount of mucus in the cytoplasm, the histological type of the gastric carcinoma was categorised into four groups. Group I: tubular differentiation - well; mucus in cytoplasm poor; group II: tubular differentiation - well; mucus in cytoplasm - rich; group III: tubular differentiation - poor; mucus in cytoplasm poor; group IV: tubular differentiation - poor; mucus in cytoplasm - rich. A study of the relation between the types of primary lesion and the mode of extension and recurrence of gastric carcinoma in 200 autopsy cases was then undertaken. In group $I$, the frequency and extent of haematogenous metastasis such as in the liver was high, while in group IV, that of lymph node metastasis, direct invasion into surrounding organ, and peritoneal dissemination were higher. In group III, which showed the intermediate mode of extension in nature to those of group I and IV, although the frequency and severity of the bone marrow metastasis was the highest. There were significant differences in the modes of development and the extent of infiltration in all groups.
\end{abstract}

Although gastric carcinoma is a malignant tumour originating from the same gastric glandular epithelium, its tissue morphology varies substantially with the individual patient and the location of the tumour in the area of the stomach - that is, whether in the pyloric, fundic, or cardiac glands. It also varies with the tissue environment of the primary and metastasised lesions in which the tumours grow and develop. As a result, there is a wide variation in the morphological future of gastric carcinoma, and there are many histological classifications of gastric carcinoma according to the criteria used. The currently used histological classification of gastric carcinoma is largely divided into two forms - that is, classification either by two histological types or by three histological types. The former classification was first introduced by Järvi et al, who proposed the metaplastic intestinal epithelial origin of gastric carcinoma' (the socalled Laüren's classification). ' This led to the theory of the two histological types, gastric and intestinal type carcinoma (differentiated and undifferentiated types), advocated by Mulligan, ${ }^{3}$ Morson, ${ }^{+}$and more recently by Nakamura et al. ${ }^{5}$ The latter classification was used in The general rules for the study of gastric cancer in surgery and pathology, edited by the Japanese Research Society for Gastric Cancer (Jpn RSGC), ${ }^{6}$ and it is consistent with Nagayo's classification by three histological types. ${ }^{7}$ The Japanese Research Society for Gastric Cancer divided the socalled common type into well differentiated tubular adenocarcinoma ( $\left.\operatorname{tub}_{1}\right)$, moderately differentiated tubular adenocarcinoma (tub $\left.{ }_{2}\right)$ and poorly differentiated adenocarcinoma (por). These were classified further into papillary adenocarcinoma (pap), mucinous adenocarcinoma (muc), and signet ring cell carcinoma (sig). In the final analysis, it appears that the difficulty of classifying $\mathrm{tub}_{2}$ in the three histological type classification by the two histological types led to the development of this complex classification by the Jpn RSGC.

When surgeons treat patients with gastric cancer, if the former classifications are used, the mode of extension after the operation, it is too difficult to select the suitable form of treatment because the modes of recurrence cannot be really identified before the actual recurrence, though these classifications are simple and useful to evaluate the preoperative extension of cancer, and to select a suitable operation procedure. On the other hand, if the latter classifications are used, it is too complicated to understand the extension of the cancer preoperatively.

The authors therefore carried out investigations in order to make a new histological classification to satisfy, even surgeons, for the requirements necessary in the treatment of patients with gastric cancer.

By using such a combination of the two histological features of gastric carcinoma, the authors attempted to classify gastric carcinoma into four groups that include undifferentiated carcinoma (ud) as well as the common type classified by the Jpn R S G C. If a discussion on the tissue origin of gastric carcinoma is set aside, this classification is basically a subtype of the two histological type classification.

NEW HISTOLOGICAL CLASSIFICATION OF GASTRIC CARCINOMA

A combination of the two histological features of gastric carcinoma - namely, (1) tubular differentiation (good or poor), and (2) mucus in the cytoplasm (rich or poor), was used as the index for the histological classification of gastric carcinoma. In other words, on the basis of (1) whether or not the differentiation of the malignant cells leads to a tubular formation, and (2) the functional status of cellular mucus productivity, gastric carcinoma was classified into the following four groups: 


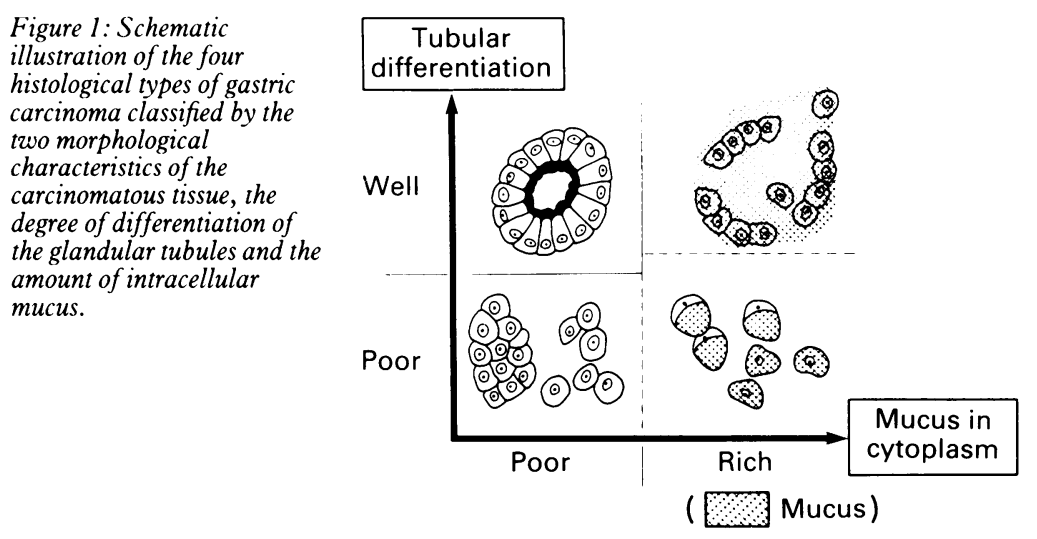

Group I: Tubular differentiation: well Mucus in the cytoplasm: poor

Group II: Tubular differentiation: well Mucus in the cytoplasm: rich

Group III: Tubular differentiation: poor Mucus in the cytoplasm: poor

Group IV: Tubular differentiation: poor Mucus in the cytoplasm: rich

Figure 1 is a schematic illustration of the above histological classification. The degree of tubular differentiation is shown on the ordinate and the amount of mucus in the cytoplasm on the abscissa. The amount of mucus in the cytoplasm was determined by the intensity of staining and the localisation of mucus in the cytoplasm, as well as by microscopically observing the histological materials prepared by alcian blue PAS double staining. As a result, tub $_{2}$ in the histological classification by the Jpn RSGC was mostly classified as group I, provided that mucus vacuoles were not present in the cytoplasm, and as group IV, provided that mucus vacuoles were present in the cytoplasm (Fig 2a, b, c, d).

The relationship between the new histological classification of gastric carcinoma and that of the Jpn RSGC is illustrated in Figure 3. Most of pap and part of tub $b_{1}$ and tub $b_{2}$ were classified as group I, while most of muc was classified as group II. Both por and ud were classified as group III, while part of sign, muc and $\operatorname{tub}_{2}$ was classified as group IV.

Using this method, the author assessed the characteristics of the mode of development of gastric carcinoma in various histological types and the results are reported in this paper.

\section{Methods}

MATERIALS

The new histological classification of gastric carcinoma described above was applied to the primary and metastasised lesions of 200 patients with gastric carcinoma (144 after gastrectomy
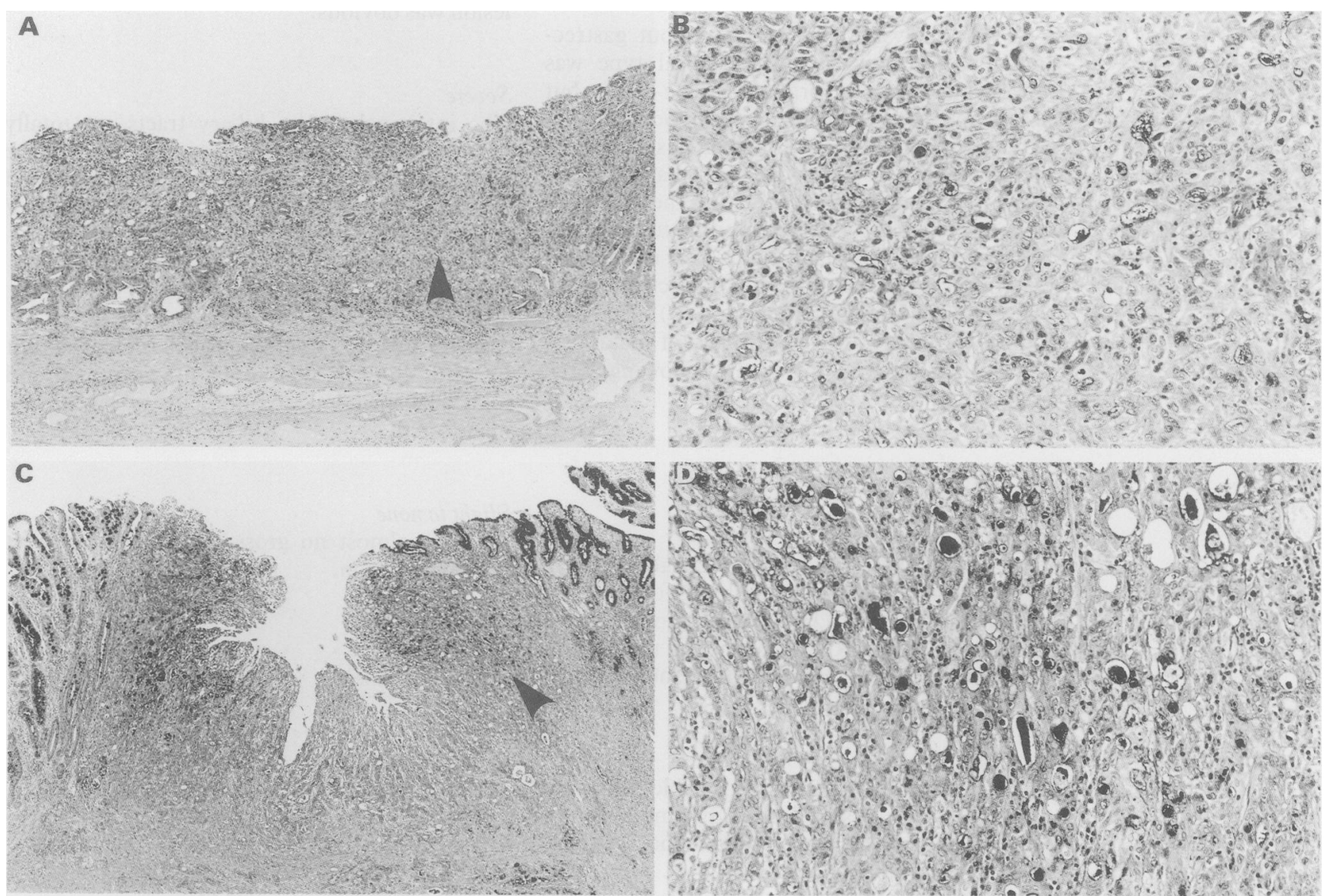

Figure 2: (a) According to the histological classification of gastric carcinoma by the fapanese Research Society for Gastric Cancer of the carcinomatous lesion (arrow) is classified as moderately differentiated tubular adenocarcinoma (tub $b_{2}$ ) (alcian blue-PAS double staining). (b) In high magnification, the carcinomatous lesion showed with arrow in Figure (a) the PAS and/or alcian blue positive deposit cannot be defined in the cytoplasm, so the authors classified this future as group I: The tubular differentiation is well and the mucus in the cytoplasm is poor (alcian blue-PAS staining). (c) According to the histological classification of gastric carcinoma by the fapanese Research Society for Gastric Carcinoma, this histological feature of carcinomatous lesion (arrow) is also classified as moderately differentiated tubular adenocarcinoma (tub $b_{2}$ ) (alcian-blue double staining). (d) In high magnification of the carcinomatous lesion showed ath arrow in (c) the $P A S$ and/or alcian blue positive mucus is apparently visible in the cytoplasm, so the authors classified this feature as group IV: the tubular differentiation is poor and the mucus in the cytoplasm is rich (alcian blue-P'PS double staining). 
Figure 3: Relationship of the new histological classification of gastric carcinoma with that of the fapanese Research Society for Gastric Cancer.

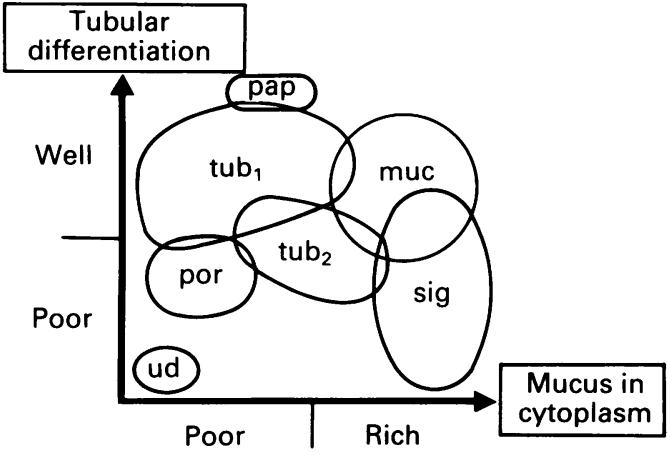

and 56 without gastrectomy) autopsied within six hours after death at the Department of Pathology, Tokyo Metropolitan Komagome Hospital over the 15 year period from 1975 to 1989. Both the primary and metastatic lesions were observed grossly in detail for the purpose of assessing the metastasis and the extent of the cancer. Specimens of the primary and metastatic lesions were prepared for histological analysis by means of haematoxylin and eosin staining and alcian blue PAS double staining, followed by a microscopic examination. The histological types were determined based on the predominant morphological findings of a primary lesion. This was done semiquantitatively, using sections in which the tumour was well represented and in which there was agreement between the observers.

In the analysis of 56 cases without gastrectomy, the predominant histological type was assessed as above for primaly gastric cancer but using those areas showing no autolytic changes.

Of the various organs to which gastric cancer metastasised, the following five areas of metastasis were mainly analysed to find the incidence and severity of metastasis according to the histological type: (1) haematogenous liver metastasis, (2) lymph node metastasis, (3) direct invasion, (4) peritoneal dissemination, and (5) metastasis to the bone marrow. The severity of metastasis was evaluated according to the following three grades:

\section{HAEMATOGENOUS LIVER METASTASIS}

Severe

The metastases have grown practically all over the lobes and seem to have been followed by hepatic insufficiency $\left(\mathrm{H}_{3}\right.$ according to the classification by the Jpn RSGC. Nearly all the hepatic tissues have been replaced by cancerous tissue.)

\section{Moderate}

Gross metastatic lesions are visible, but are unlikely to have caused an impairment of the hepatic function $\left(\mathrm{H}_{1-2}\right.$ according to the classification by the Jpn RSGC).

\section{Slight to none}

There is no gross evidence of metastasis. If present, it is detected by a histological examination $\left(\mathrm{H}_{0}\right.$ or $\mathrm{h}_{0}$ and $\mathrm{h}_{1}$ according to the classification by the Jpn RSGC)

\section{LYMPH NODE METASTASIS}

Severe

Metastases have spread to the distant lymph nodes such as the Virchow, auxiliary and/or inguinal lymph nodes, including other superficial lymph nodes outside the abdominal and thoracic cavities.

\section{Moderate}

Metastases have spread to the regional lymph nodes in the abdominal cavity, lymph nodes surrounding the aorta, and those in the thoracic cavity.

\section{Slight to none}

Metastases are confined to the regional lymph nodes in the abdominal cavity and have not extended to the lymph nodes surrounding the aorta. (Clinically, lymph node metastases are surgically resectable.)

\section{DIRECT INVASION}

Strictly speaking, to differentiate between direct invasion and peritoneal dissemination is impossible in many cases (particularly those in group IV). This section deals with the cases where the direct and continuous invasion of the primary lesion was obvious.

\section{Severe}

The intestinal and/or biliary tracts are totally obstructed by a direct tumour invasion (all of the patients are considered to have developed clinical symptoms of intestinal obstruction and/ or obstructive jaundice)

\section{Moderate}

A direct invasion to other organs is evident, but marked clinical symptoms of intestinal and biliary obstruction are unlikely to have been manifested.

\section{Slight to none}

There is almost no gross evidence of a direct tumour invasion.

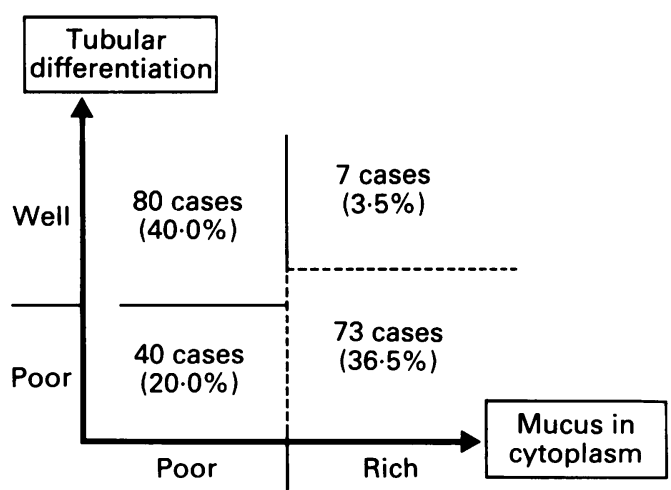

Figure 4: Patient numbers in each of the histological types of primary lesion in 200 autopsy cases classified by the new histological classification. 


\section{PERITONEAL DISSEMINATION}

\section{Severe}

As a result of severe dissemination, the visceral and parietal peritoneum form a mass covered with a thick plaster like fibrous cancer tissue.

\section{Moderate}

Gross peritoneal dissemination is visible, but it has not diffused all over the intestinal wall and the peritoneum. Although disseminated metastatic nodes are present, no clinical symptoms of intestinal and biliary stenosis are unlikely to have been manifested.

\section{Slight to none}

There is no gross evidence of peritoneal dissemination. If present, it is detected by a histological examination.

\section{METASTASIS TO THE BONE MARROW}

Severe

Numerous gross metastatic lesions are seen, replacing nearly all of the bone marrow.

\section{Moderate}

Gross metastatic lesions are limited to several areas of the bone marrow.

\section{Slight to none}

There is no gross evidence of metastatic lesions. If present, they are detected by a histological examination.

\section{OTHER METASTASIS}

Although metastatic lesions in other organs, including the lung, ovary, etc, were also examined histologically, the results are omitted from this paper.

\section{Results}
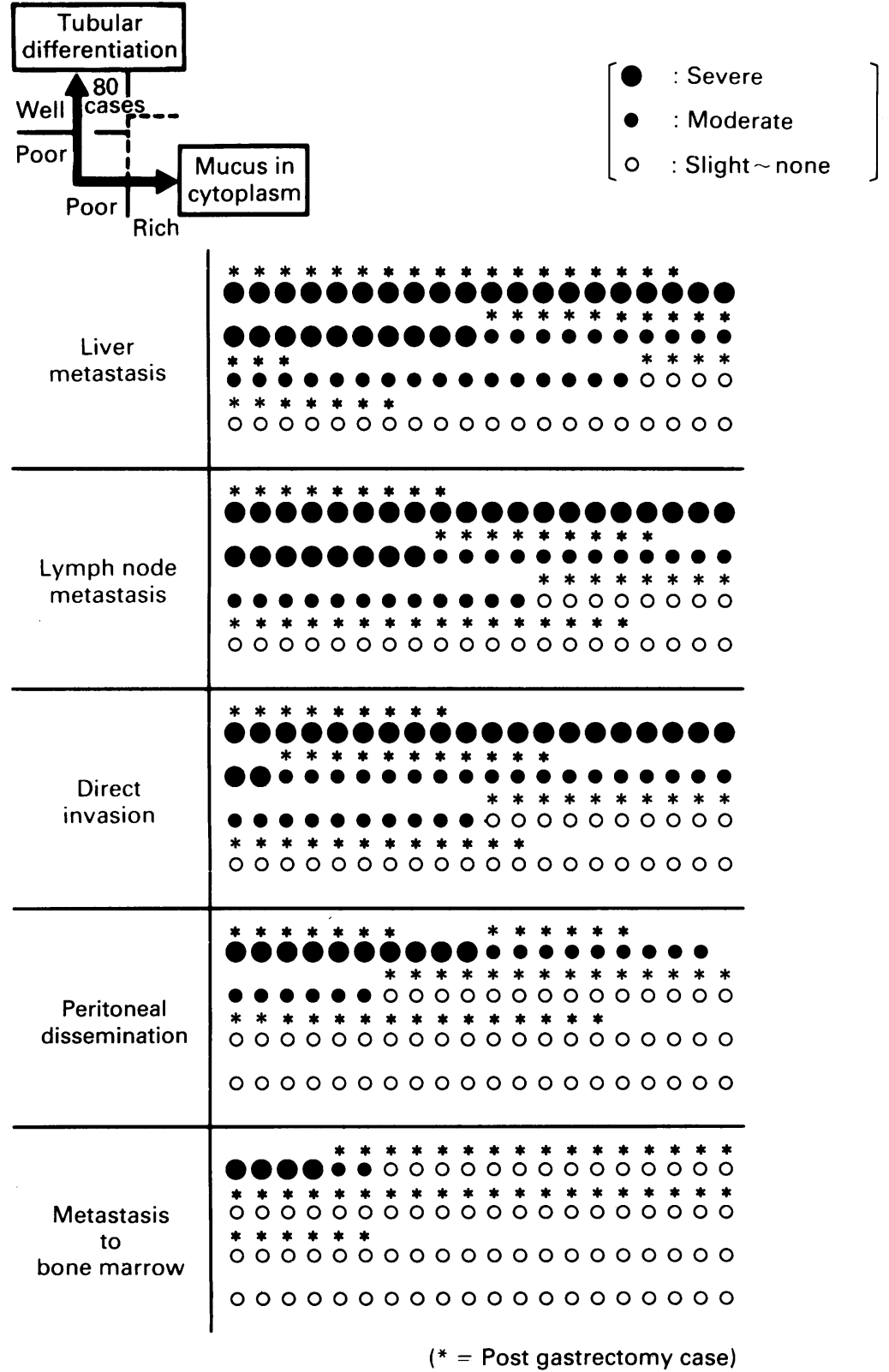

Figure 5: Mode of development and recurrence in 80 autopsy cases, histologically classified in group I: the tubular differentiation is well and the mucus in the cytoplasm is poor.

\section{DIFFERENCES OF THE MODE OF SPREADING OF GASTRIC CARCINOMA CONCERNED WITH THE HISTOLOGICAL TYPES}

According to the new histological classification $(40 \cdot 0 \%)$ were classified as group I, seven $(3 \cdot 5 \%)$ as group II, $40(20.0 \%)$ as group III, and 73 (36.5\%) as group IV (Fig 4). The mode of development of gastric carcinoma in the histological groups is analysed below.

GROUP I (Fig 5)

Of the 80 cases in group I, $42(52 \cdot 5 \%)$ had undergone a gastrectomy (indicated by an asterisk $\left(^{\star}\right)$ in the Figure), while the remaining $38(47 \cdot 5)$ had not undergone a gastrectomy.

\section{Liver metastasis}

Moderate to severe haematogenous liver metastases were observed in 56 cases $(70.0 \%$ ), of which $30(37 \cdot 5 \%)$ had severe metastases.

\section{Lymph node metastasis}

Because the subjects were all autopsy cases, the incidence of severe lymph node metastases was naturally high. Moderate to severe metastases were observed in 52 cases $(65.0 \%)$, of which 28 $(35.0 \%)$ had severe metastases. In $28(35.0 \%)$, lymph node metastasis was found to some extent or not detected. Upon a gross examination, it was found that the metastatic nodes in this group were larger and more configurated than those observed in the other groups.

\section{Direct invasion}

Moderate to severe direct invasion was observed in 50 cases $(62 \cdot 5 \%)$, of which $22(27 \cdot 5 \%)$ had a severe invasion. Direct invasion was found to some extent or not detected in remaining 30 (37.5\%). of gastric carcinoma, of the 200 autopsy cases, 80 
Peritoneal dissemination

In $54(67 \cdot 5 \%)$, dissemination was found to some extent or not detected. Moderate to severe peritoneal dissemination was observed in only 26 cases $(32 \cdot 5 \%)$, of which $10(12 \cdot 5 \%)$ had severe dissemination.

\section{Metastasis to the bone marrow}

Moderate to severe bone marrow metastases were observed in six cases $(7 \cdot 5 \%)$, of which four $(5.0 \%)$ had severe metastases. In remaining 74 $(92.5 \%)$ bone marrow metastasis was found to some extent or not detected.

\section{GROUP II}

As only seven cases $(3 \cdot 5 \%)$ were classified as group II, these were not analysed in the present study. As soon as enough cases are found, however, the findings will be analysed.

\section{GROUP III (Fig 6)}

Forty cases $(20.0 \%)$ were classified as group III, of which $20(50 \%)$ had undergone a gastrectomy and remaining $20(50 \%)$ had not.
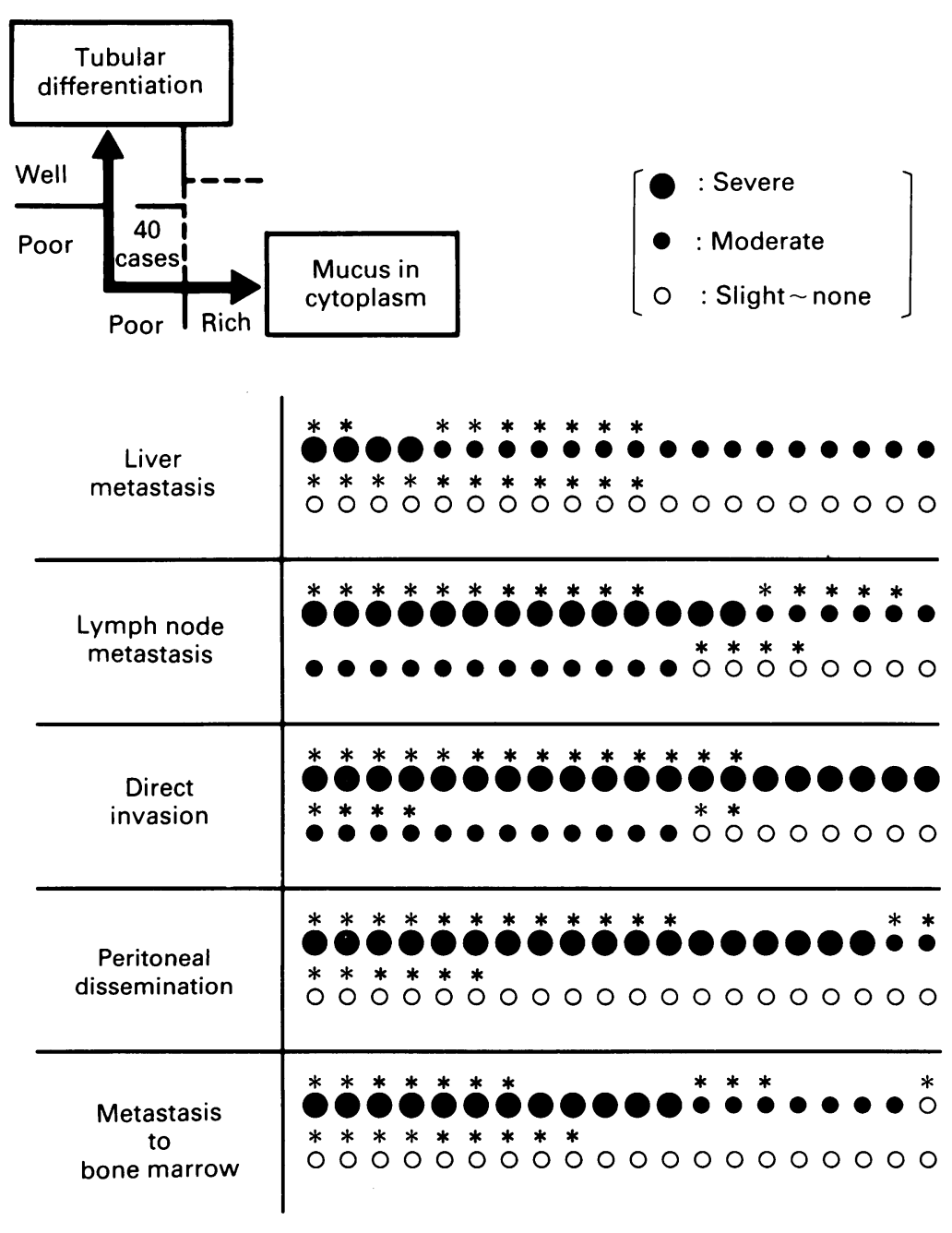

$1^{*}=$ Post gastrectomy case)

Figure 6: Mode of development and recurrence in 14 autopsy cases, histologically classified in group III: the tubular differentiation is poor and the mucus in the cytoplasm is also poor.

\section{Liver metastasis}

Moderate to severe haematogenous liver metastases were observed in 20 cases $(50.0 \%$ ), of which four $(10.0 \%)$ had severe metastases. Liver metastasis was found to some extent or not detected in the remaining $20(50 \cdot 0 \%)$.

\section{Lymph node metastasis}

Moderate to severe metastases were observed in 32 cases $(80.0 \%)$, of which $14(35 \cdot 0 \%)$ had severe metastases. In the remaining eight $(20 \cdot 0 \%)$ lymph node metastasis was found to some extent or not detected.

\section{Direct invasion}

Moderate to severe direct invasion was observed in 32 cases $(80.0 \%)$, of which $20(50.0 \%)$ had a severe invasion. A direct invasion was found to some extent or not detected in eight $(20.0 \%)$.

\section{Peritoneal dissemination}

Moderate to severe peritoneal dissemination was observed in 20 cases $(50.0 \%)$, of which 18 $(38.9 \%)$ had severe dissemination. Peritoneal dissemination was found to some extent or not detected at all in the remaining $20(50 \cdot 0 \%)$.

\section{Metastasis to the bone marrow}

Moderate to severe bone marrow metastases were observed in 19 cases $(47 \cdot 5 \%)$, of which 12 $(30.0 \%)$ had severe metastases. In the remaining $21(52 \cdot 5 \%)$ bone marrow metastasis was found to some extent or not detected.

GROUP IV (Fig 7)

Seventy three cases $(36.5 \%)$ were classified as group IV, of which $53(72 \cdot 6 \%)$ had undergone a gastrectomy and $20(27 \cdot 4 \%)$ had not.

\section{Liver metastasis}

Moderate haematogenous liver metastases were observed in 10 cases $(13.7 \%$ ), but no cases of severe metastases were observed. In the remaining $63(86 \cdot 3 \%)$ liver metastasis was found to some extent or not detected.

\section{Lymph node metastasis}

Moderate to severe metastases were observed in 64 cases $(87 \cdot 7 \%)$, of which $50(68 \cdot 5 \%)$ had severe metastases. Only in the remaining nine $(12 \cdot 3 \%)$ lymph node metastasis was found to some extent or not detected.

As compared with group I, the metastatic nodes in this group were small and confluent, accompanied by peritoneal dissemination.

\section{Direct invasion}

Moderate to severe direct invasion was observed in 70 cases $(90.3 \%)$, of which $58(79.4 \%)$ had a severe invasion. In the remaining three $(4 \cdot 1 \%)$, a direct invasion was found to some extent or not detected. 


\section{Peritoneal dissemination}

Moderate to severe peritoneal dissemination was observed in 68 cases $(93 \cdot 1 \%)$, of which 63 $(86 \cdot 3 \%)$ had severe dissemination. In the remaining five $(6 \cdot 9 \%)$, peritoneal dissemination was found to some extent or not detected.

\section{Metastasis to the bone marrow}

Bone marrow metastasis was found to some extent or not detected in $55(75 \cdot 3 \%)$. Moderate to severe bone marrow metastases were observed in 18 cases $(24 \cdot 7 \%)$, of which $14(19 \cdot 2 \%)$ had severe metastases.

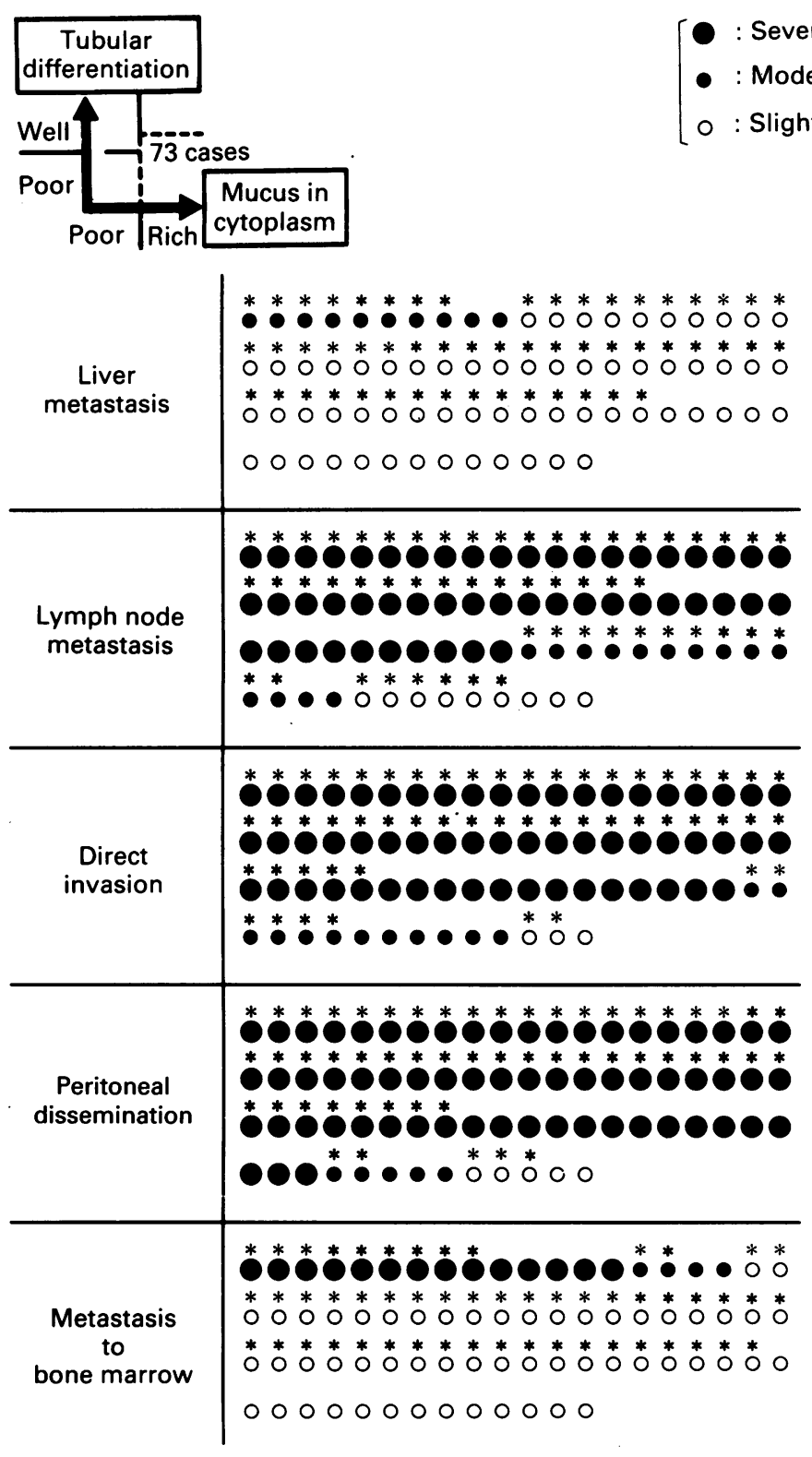

$1^{*}=$ Post gastrectomy case)

Figure 7: Mode of development and recurrence in 73 autopsy cases, histologically classified in group IV: the tubular differentiation is poor and the mucus in the cytoplasm is rich. $A$ combination of two morphological features, the degree of tubular differentiation and the content of mucus in the cytoplasm, was used to classify the histological types of gastric carcinoma into four groups. The mode of development and recurrence in autopsy cases was assessed according to the histological types of primary lesion. As a result, the characteristic mode of development and recurrence could be observed in the four histological groups. Furthermore, there were statistically significant differences in the incidence and severity of the metastases among the groups.
COMPARISON BY MODE OF DEVELOPMENT AMONG THE HISTOLOGICAL GROUPS AND THE STATISTICAL ANALYSIS OF THE CHARACTERISTICS

The mode of development of gastric carcinoma, as determined by the incidence and severity of various types of metastasis, were compared among the histological groups. The results were analysed statistically using the Kruskal-Wallis $\mathrm{H}$-test in an attempt to clarify the characteristics of the mode of development in the histological groups and to assess the significance of the new histological classification. There were 97 patients in groups I, III, and IV. The seven patients in group II were excluded from the analysis in the present study because of the limited number. The results of the analysis are shown according to the type of metastasis.

\section{Liver metastasis}

The H-test analysis indicated significant differences in the incidence and severity of liver metastasis among the three histological groups $(p<0.01)$. The groups in the order of the highest incidence and severity were I, III, and IV.

\section{Lymph node metastasis}

There were also significant differences in the incidence and severity of lymph node metastasis among the histological groups $(p<0.05)$. As autopsy patients usually have highly advanced cancer (terminal cancer), the incidence of lymph node metastasis was naturally high. The groups in the order of the highest incidence and severity of lymph node metastasis were IV, III, and $I$.

\section{Direct invasion}

There were also significant differences in the incidence and severity of direct invasion among the histological groups $(\mathrm{p}<0.01)$. The groups in the order of the highest incidence and severity were IV, III, and I.

\section{Peritoneal dissemination}

There were significant differences in the incidence and severity of peritoneal dissemination among the histological groups $(p<0 \cdot 01)$. The groups in the order of the highest incidence and severity were IV, III, and I.

\section{Metastasis to the bone marrow}

There were significant differences in the incidence and severity of bone marrow metastasis among the histological groups $(p<0 \cdot 01)$. The groups in the order of the highest incidence and severity were III, IV, and I.

The statistical significance of the differences among the histological groups was further analysed using Wilcoxon's two sample test (t test). There were significant differences between group I and III $(\mathrm{p}<0.01)$ and group I and IV $(p<0.05)$. No significant difference was observed, however, between groups III and IV $(\mathrm{p}<0 \cdot 10)$. 


\section{Discussion}

HISTOLOGICAL CLASSIFICATION OF GASTRIC CARCINOMA ACCORDING TO THE TUBULAR DIFFERENTIATION AND MUCUS PRODUCTION Histologically, the criteria for the histological classification of tumours has been based on the morphological characteristics of (1) the organ where carcinoma originated and (2) the histogenesis, or (3) the degree and the characteristics of the atypical cells.

The histological classification of gastric carcinoma such as Laüren's classification and Nakamura's classification in Japan is based on the findings of the proposed histogenesis. On the other hand, the classification by the Jpn RSGC or Ohta (Ohta's classification ${ }^{8}$ ) uses the degree and features of the atypical cells.

The histological classification examined in this paper uses a combination of the two characteristics of gastric cancer, the structural feature of tubular differentiation, and the functional status of mucus production.

The use of tubular differentiation as an index of the structure of cancer tissue is unlikely to raise any questions. The use of the mucus content in the cytoplasm as an index of the functional characteristic of gastric cancer cells, however, may be debatable. Because signet ring cell carcinoma proliferates in. a disorganised fashion and does not form glandular tubules, the formed mucus cannot be drained, this causes mucus to accumulate in the cytoplasm. According to Nagayo, an electron microscopic study of signet ring cells appearing small and containing a relatively limited amounts of mucus as seen under a light-microscopic examination (type I according to his classification) revealed mucus secretion, the presence of abundant organelles in the cells, and well developed microvilli on the surface of the cells. ${ }^{\text {? }}$

It is therefore too short sighted to merely conclude that the accumulation of mucus in signet ring cell adenocarcinoma is attributable to the absence of tubular differentiation that hampers the drainage of the mucus. For this reason, we chose the amount of mucus in the cytoplasm as an index of the functional status of gastric cancer and used it in combination with the structural characteristic of tubular differentiation for the histological classification of gastric carcinoma. This method must be assessed further in a greater number of patients.

MODE OF DEVELOPMENT OF GASTRIC CARCINOMA ACCORDING TO THE HISTOLOGICAL TYPE

To date, a number of studies have been conducted on the mode of development of gastric carcinoma in autopsy cases.9-1+ Basically, the results of the present study are not entirely different from those of other studies. Nevertheless, there has been gradual progress in various methods of treatment in cancer therapy and, as a result, the clinical course of gastric carcinoma up to the time of death - that is, the mode of development and recurrence has changed in a subtle way.

Furthermore, it is apparent from the results of the statistical analysis of the current study, that the classification of primary lesions by the new histological classification of gastric carcinoma is useful in predicting the conditions of the metastases observed during an autopsy. This means that the method in question has a high rate of predictability for the mode of development and recurrence of gastric carcinoma if applied to the classification of the histological features of a stomach biopsy and resected specimens. Furthermore, the use of the new histological classification seems to be helpful in determining the site of examination, the necessary precautions, and the guidelines for treatment during the follow up period. Thus far, the authors have not compared this classification with other classification methods, but a comparative study will be conducted in the future.

The authors are deeply grateful to Takanori Mochizuki, MD, former Director of the Department of Pathology, Tokyo Metropolitan Komagome Hospital, for his guidance in conducting the present study and to Tadashige Murakami, MD ex-Professor of the First Department of Surgery, School of Medicine, Tokyo Medical and Dental University, for his words of encouragement in this study.

1 Järvi O, Läuren P. On the role of heterotopias of the intestinal epithelium in the pathogenesis of gastric cancer. Acta Patho Microbiol Scand 1951; 29: 26-44.

2 Laüren P. The two histological main types of gastric carcinoma. Acta Pathol Microbiol Scand 1965; 64: 31-49.

3 Mulligan RM, Rember RR. Histogenesis and the biological behavior of gastric carcinoma. Arch Pathol 1954; 58 : 1-25.

4 Morson BC Carcinoma arising from areas of intestinal metaplasia in the gastric mucosa. Br F Cancer 1955; 9: 377-85.

5 Nakamura K, Sugano H, Takagi K, Kumakura K Histogenesis of carcinoma of the stomach with special reference to 50 primary microcarcinomas: light-/Electronmicroscopic and statistical studies. $\mathcal{F p n} \mathcal{J}$ Cancer Clin 1969; microscopic

6 Japanese Research Society For Gastric Cancer. The Genera rules for the study of gastric cancer in surgery and pathology. 11 th ed. Tokyo: Kanehara Publishing, 1985

7 Nagayo T. Igan Soshikihassei ni kansuru Soshikigakuteki Jikkenteki Kenkyu. Transactiones Societatis Pathologica Faponicae 1976; 65: 3-25.

8 Ohta K. Igan no Hassei. Transactiones Societis Pathologica Faponicae 1965; 53: 1-16.

9 Imai T. The growth of human carcinoma - a morphological analysis. Fukuoka Ishi 1954; 45: 45-102.

10 Furuno H. Pathological anatomical studies on the matastasis of the stomach carcinoma. Igaku Kenkyu $S$ 32;27: 1496-151.

11 Araki T. A patho-morphological study on the distribution of the metastasis of gastric carcinoma - especially the clinicothe metastasis of gastric carcinoma - especially the clinico-
pathological consideration for the cases of gastric carcinoma pathological consideration for the cases of gastric carcinoma not identifiable clinically. Transactio
logicae faponicae 1959; 48: 757-98.

12 Tanaka T. Pathological studies on the lesion of gastric cance and the distribution of its metastases - a comparative study between gastrectomied and non-gastrectomied cases. Show Igakukai-shi 1963; 23: 362-87.

13 Soejima $\mathrm{K}$. The recurrence of gastric cancer - a review of 2 cases examined at autopsy. Fukuoka Igaku-zasshi 1963; 55: $410-30$.

14 Ishii T, Ikegami N, Hosoda Y, Koide O, Kaneko M. The biological behavior of gastric cancer. F Pathol 1981; 134: 97 115. 